\title{
Prevention and regression of non-alcoholic steatohepatitis (NASH) in a rat model by metabosartan, telmisartan
}

\author{
HIRONORI NAKAGAMI ${ }^{1}$, MARIANA KIOMY OSAKO ${ }^{2,3}$, FUTOSHI NAKAGAMI $^{2,3}$, TAKASHI SHIMOSATO ${ }^{4}$, \\ NORIKO MINOBE ${ }^{4}$, TOSHINORI MORITANI ${ }^{4}$, MUNEHISA SHIMAMURA $^{2}$, TAKASHI MIYAKE $^{2}$, \\ HIDEO SHIMIZU ${ }^{2,3}$, YASUSHI TAKEYA ${ }^{3}$ and RYUICHI MORISHITA ${ }^{2}$
}

Departments of ${ }^{1}$ Gene Therapy Science, ${ }^{2}$ Clinical Gene Therapy, and ${ }^{3}$ Geriatric Medicine, Osaka University Graduate School of Medicine, Suita, Osaka 565-0871; ${ }^{4}$ Research Department, Nissei Bilis Co. Ltd., Koka, Shiga 528-0052, Japan

Received February 5, 2010; Accepted April 6, 2010

DOI: $10.3892 /$ ijmm_00000488

\begin{abstract}
The favorable metabolic effects of telmisartan have been attributed to its angiotensin II receptor blockade and action as a partial agonist of peroxisome proliferator-activated receptor (PPAR) $-\gamma$. We previously reported that administration of telmisartan markedly inhibited lipid accumulation in the liver in mice fed a high-fat diet. In the present study, we further examined the protective effect of telmisartan in a nonalcoholic steatohepatitis (NASH) model induced by feeding Wistar rats an L-methionine- and choline-deficient (MCA) diet. In the first experiment, rats were fed an MCA diet for 8 weeks with or without telmisartan $(3 \mathrm{mg} / \mathrm{kg} / \mathrm{day})$. Liver fibrosis was observed by Masson trichrome staining, and cotreatment was shown to attenuate liver fibrosis. In the second experiment, Wistar rats were fed an MCA diet for 20 weeks, and telmisartan $(3 \mathrm{mg} / \mathrm{kg} /$ day $)$ was administered during weeks 0-20 as a preventive model or weeks $8-20$ as a therapeutic model. As a result, telmisartan administration in both models significantly attenuated liver fibrosis and an increase in serum AST. Of importance, the HGF concentration in the liver was significantly increased in the telmisartan-treated group. Overall, telmisartan showed a potential action to improve NASH induced by an MCA diet, possibly due to increased HGF production through partial agonist of PPAR- $\gamma$. These favorable characteristics of telmisartan as a partial agonist of PPAR- $\gamma$ may provide a benefit in the treatment of metabolic syndrome beyond its blood pressure-lowering effect.
\end{abstract}

\section{Introduction}

Telmisartan, an angiotensin (Ang) type I receptor (AT1R) blocker (ARB), has been widely used for the treatment of

Correspondence to: Professor Ryuichi Morishita, Department of Clinical Gene Therapy, Osaka University Graduate School of Medicine, 2-2 Yamada-oka, Suita, Osaka 565-0871, Japan

E-mail: morishit@cgt.med.osaka-u.ac.jp

Key words: telmisartan, non-alcoholic steatohepatitis, HGF hypertension and hypertension-related cardiovascular endorgan damage (1). It has also been identified as a unique, moderately potent, selective partial agonist of peroxisome proliferator-activated receptor- $\gamma$ (PPAR- $\gamma$ ) $(2,3)$, which plays an important role in regulating carbohydrate and lipid metabolism, leading to the improvement of insulin sensitivity, reduced triglyceride level and decreased risk of atherosclerosis (4-6). For example, telmisartan administration caused significant attenuation of weight gain and reduced glucose, insulin and triglyceride levels in male Sprague-Dawley rats fed a high-fat, high-carbohydrate diet, and also improved insulin sensitivity in diet-induced obese mice without weight gain $(2,7)$, which might involve inhibition of renin-angiotensin system activity and PPAR- $\gamma$ activation.

We previously demonstrated that administration of telmisartan improved endothelial dysfunction to a greater extent when compared with that by losartan, probably due to the effects on vascular HGF level (8). Importantly, we also demonstrated that telmisartan attenuated fatty liver in this study; however, detailed analysis of the effect of telmisartan on the liver has not yet been reported. Thus, in the present study, we examined the effect of telmisartan in a non-alcoholic steatohepatitis (NASH) model induced by feeding wild Wistar rats an L-methionine- and choline-deficient (MCA) diet. We showed a beneficial effect of telmisartan on liver fibrosis in two different experiments, a preventive and a therapeutic model.

\section{Materials and methods}

Animals and diets. This study was approved by the Ethics Committee for Animal Experiments of Osaka University Graduate School of Medicine. Male Wistar rats were obtained from Jackson Laboratory (Bar Harbor, ME). Rats (age 8 weeks) were fed an MCA diet (\#518810) or a control diet (\#518811) (both from Dyets Inc.) with or without oral administration of telmisartan ( $3 \mathrm{mg} / \mathrm{kg} /$ day $)$ for 8 or 20 weeks. Rats had free access to water and food during the experimental periods.

Body weight was measured every week, and liver weight was measured for 20 weeks after the start of the MCA diet. Serum AST (GOT), ALT (GPT), ALP, total protein and total 
A

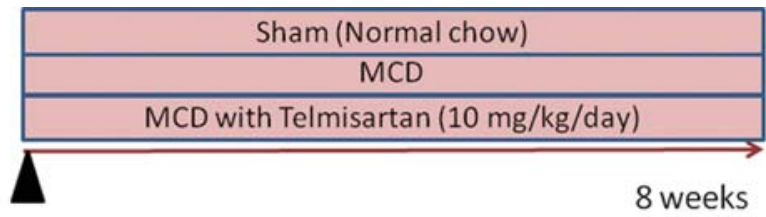

B

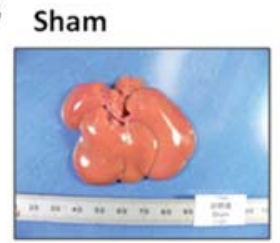

MCD
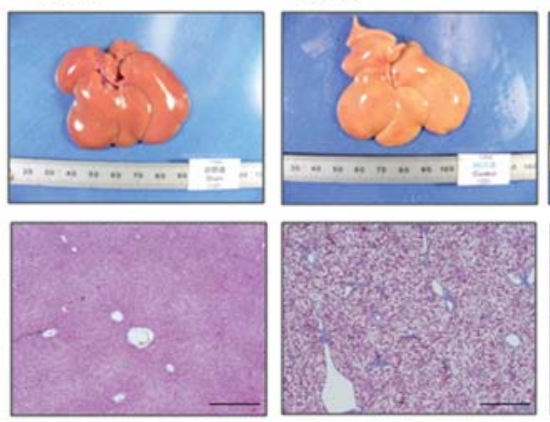

MCD+Telmisartan
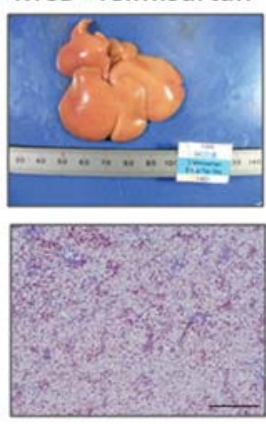

C

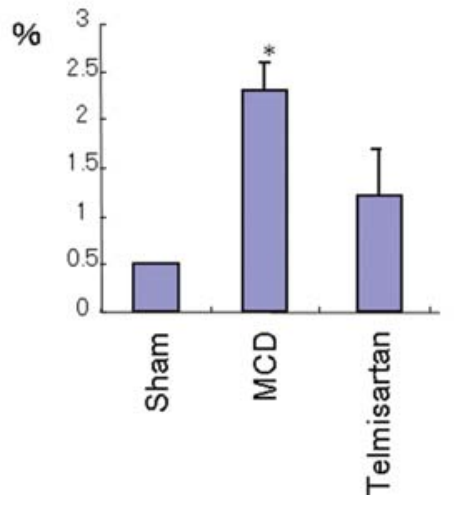

Figure 1. Acute NASH model induced by an MCA diet for 8 weeks. (A) The rats were divided into three groups: Sham, rats fed normal chow $(n=5)$; MCA, rats fed an MCA diet (n=5); MCA + telmisartan, rats fed an MCA diet and co-administered telmisartan $(n=5)$. (B) Upper panel, representative images of liver; lower panel, Masson trichrome staining in the liver (scale bar, $500 \mu \mathrm{m}$ ). (C) The fibrosis area was quantified by calculating the positive area (blue color) with Masson trichrome staining. ${ }^{*} \mathrm{P}<0.05$ compared to the Sham group.

cholesterol were measured at $0,4,8,16$ and 20 weeks during the MCA diet.

Study protocol. The first experiment was performed in an acute NASH model induced by an MCA diet for 8 weeks. Telmisartan was obtained from Boehringer-Ingelheim. The rats were divided into three groups: i) rats fed normal chow (Sham, n=5), ii) rats fed an MCA diet (MCA, n=5) and iii) rats fed an MCA diet and orally co-administered telmisartan (3 mg/kg/day) (MCA + telmisartan, $\mathrm{n}=5$ ).

The second experiment was performed in a chronic NASH model induced by an MCA diet for 20 weeks. The rats were divided into four groups: i) rats fed normal chow (Sham, $n=5)$, ii) rats fed an MCA diet (MCA, n=5), iii) rats fed an MCA diet and orally co-administered telmisartan ( $3 \mathrm{mg} / \mathrm{kg} / \mathrm{day}$ ) for 20 weeks (MCA + telmisartan weeks 0-20, $\mathrm{n}=5)$ and iv) rats fed an MCA diet and orally co-administered telmisartan $(3 \mathrm{mg} / \mathrm{kg} /$ day) from week 8 to $20(\mathrm{MCA}+$ telmisartan weeks 8-20, $\mathrm{n}=5$ ).

Evaluation of liver fibrosis. Extracted liver samples were photographed, and liver weight was measured after removal

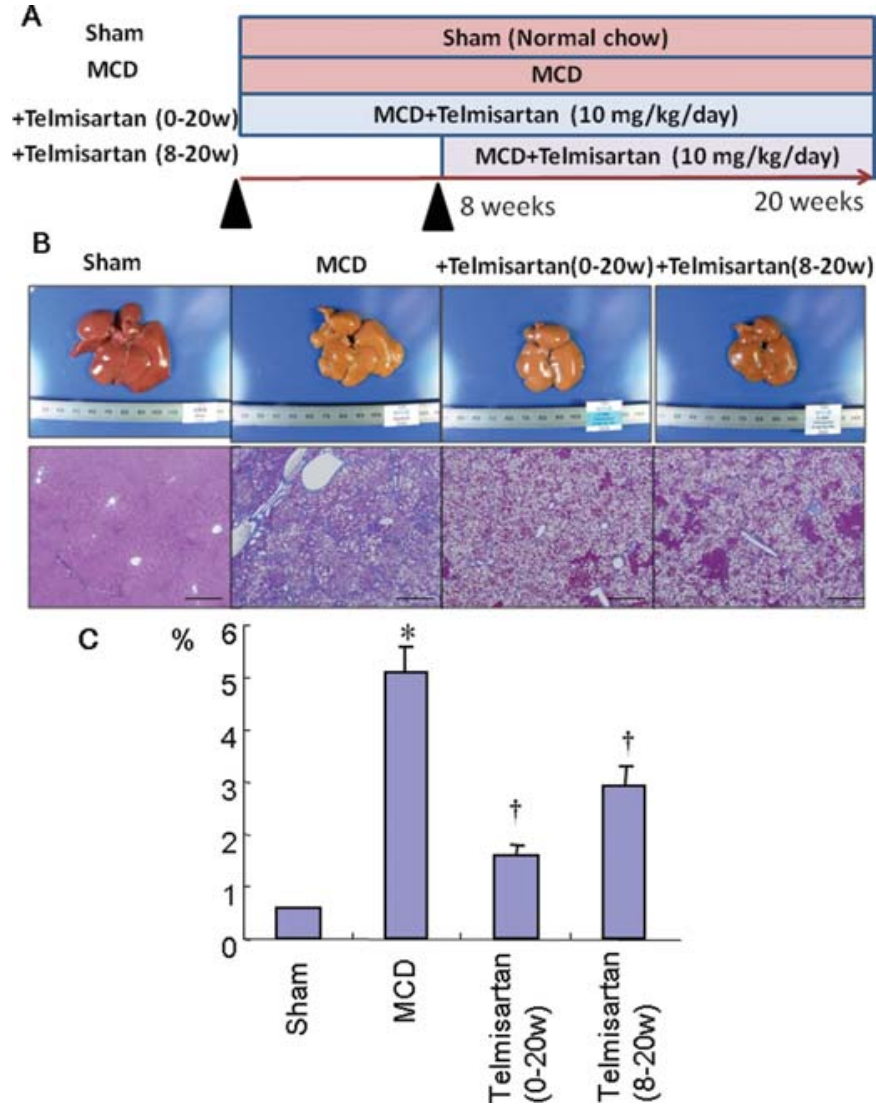

Figure 2. Chronic NASH model induced by an MCA diet for 20 weeks. (A) The rats were divided into four groups: Sham, rats fed normal chow $(n=5)$; MCA, rats fed an MCA diet $(n=5) ;$ MCA + telmisartan weeks 0-20, rats fed an MCA diet and co-administered telmisartan for 20 weeks $(n=5)$; and MCA + telmisartan weeks 8-20, rats fed an MCA diet and coadministered telmisartan from week 8 to $20(n=5)$. (B) Upper panel, representative images of liver; lower panel, Masson trichrome staining in the liver (scale bar, $500 \mu \mathrm{m}$ ). (C) The fibrosis area was quantified by calculating the positive area (blue color) with Masson trichrome staining ${ }^{*} \mathrm{P}<0.05$ compared to the Sham group. ${ }^{\dagger} \mathrm{P}<0.05$ compared to the MCA group.

of water and attached tissues. Half of the liver tissue was fixed with $10 \%$ formaldehyde, and each slide was stained with $\mathrm{H} \& \mathrm{E}$ or Masson trichrome. The area of fibrosis was evaluated by positive staining with Masson trichrome measured using Win Roof (version 5.5, Mitsuya-Shoji) and shown as the percentage of the total area.

The other half of the liver was homogenized for HGF and TGF- $ß 1$ ELISA (R\&D Systems, Minneapolis, MN), exactly as described by the manufacturer.

Statistical analysis. All values are expressed as the mean \pm SD. Data were compared using ANOVA followed by the Dunnett's test for pair-wise comparisons against the control and by the Tukey's test for multiple comparisons. All statistical analysis was performed using Stat-View 5.0 software (SAS Institute, Inc., Cary, NC). Values of $\mathrm{P}<0.05$ were considered to indicate statistical significance.

\section{Results}

In the first experiment, rats were fed an MCA diet for 8 weeks to induce liver fibrosis and then co-administered 


\section{A}
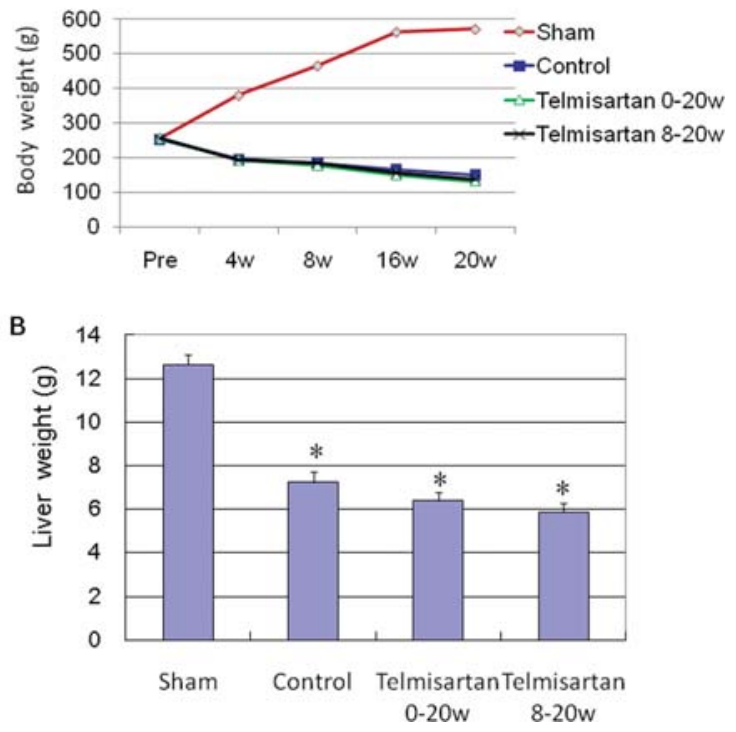

Figure 3. Body weight and liver weight in the MCA model. (A) Body weight at $0,4,8,16$ and 20 weeks and (B) liver weight at 20 weeks in the four groups: Sham, rats fed normal chow $(n=5)$; MCA, rats fed an MCA diet $(n=5)$; MCA + telmisartan weeks 0-20, rats fed an MCA diet and coadministered telmisartan for 20 weeks $(n=5)$; and MCA + telmisartan weeks $8-20$, rats fed an MCA diet and co-administered telmisartan from week 8 to $20(n=5) .{ }^{*} \mathrm{P}<0.01$ compared to the high-fat diet group.

telmisartan (Fig. 1A). Without telmisartan treatment, the color of the liver changed to yellow, and liver fibrosis was observed by Masson trichrome staining. In the telmisartan-treated group, the color of the liver did not change to yellow, and the fibrosis area was reduced (Fig. 1B and C). Based on these results, we designed the next experiment.

In the second experiment, the rats were fed an MCA diet for 20 weeks, and the telmisartan-co-treated group was divided into two groups: co-administration of telmisartan for 20 weeks as a preventive model and co-administration of telmisartan from week 8 to 20 (12 weeks) as a therapeutic model (Fig. 2A). The MCA diet for 20 weeks markedly changed the color of the liver to yellow and induced liver fibrosis upon Masson trichrome staining, whereas telmisartan treatment prevented the yellow color change of the liver and significantly reduced liver fibrosis, not only in the preventive model, but also in the therapeutic model (Fig. 2B and C). Rats fed the MCA diet for 20 weeks showed a marked decrease in body and liver weight, an increase in serum GOT level and a decrease in total protein and total cholesterol levels, suggesting liver dysfunction. In this model, co-administration of telmisartan did not show any effect on body and liver weight (Fig. 3A and B). However, serum GOT at week 16 and 20 after the start of the MCA diet was decreased by treatment with telmisartan in both co-treatment models (Fig. 4A). Serum total protein and total cholesterol also showed a similar tendency to GOT in both models (Fig. 4B and C). These results suggest that co-administration of telmisartan improved liver function in both the preventive and therapeutic models.

In our previous study, telmisartan significantly increased local HGF expression in the aorta (8). Thus, we measured HGF and TGF- $\$ 1$ production in the liver, as anti-fibrotic and pro-fibrotic factors, respectively, to analyze how telmisartan
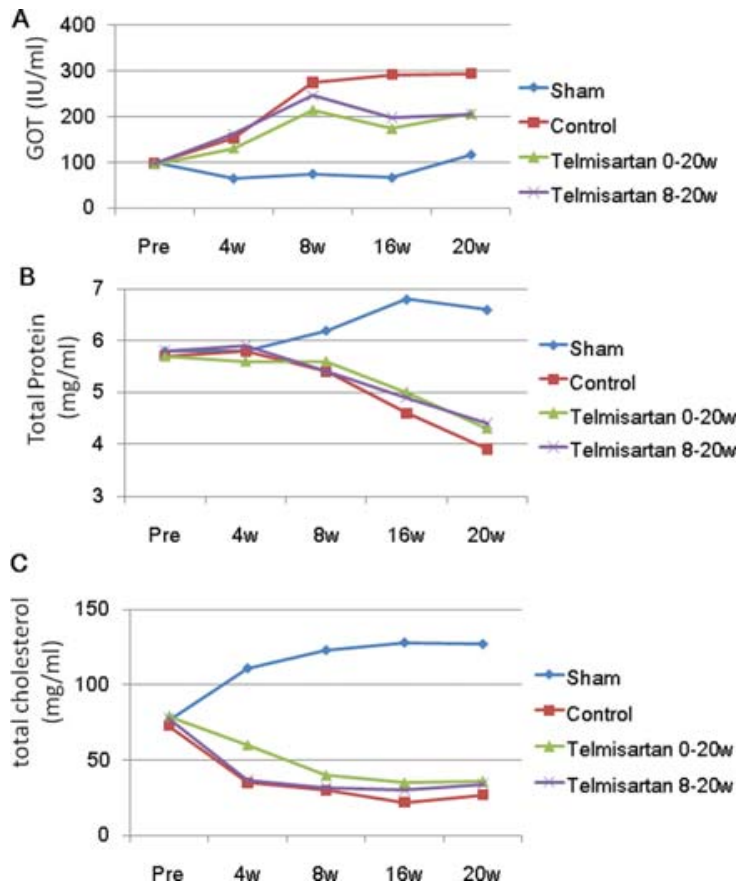

Figure 4. Serum GOT, total protein, and total cholesterol level at 0, 4, 8, 16 and 20 weeks after start of an MCA diet in the four groups: Sham, rats fed normal chow $(n=5)$; MCA, rats fed an MCA diet $(n=5)$; MCA + telmisartan weeks $0-20$, rats fed an MCA diet and co-administered telmisartan for 20 weeks $(n=5)$; and MCA + telmisartan weeks 8-20, rats fed an MCA diet and co-administered telmisartan from week 8 to $20(n=5)$.

A
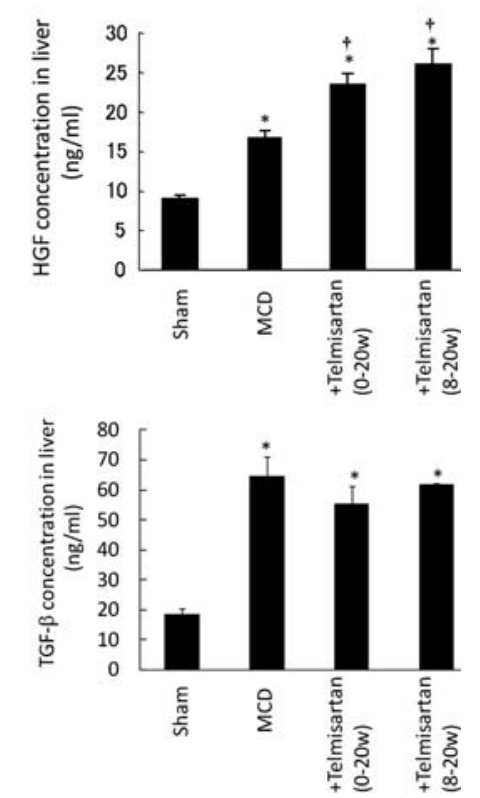

Figure 5. HGF and TGF- 31 production in the liver at 20 weeks after the start of an MCA diet in the four groups: Sham, rats fed normal chow $(n=5)$; MCA, rats fed an MCA diet $(n=5)$; MCA + telmisartan weeks 0-20, rats fed an MCA diet and co-administered telmisartan for 20 weeks $(n=5)$; and MCA + telmisartan weeks 8-20, rats fed an MCA diet and co-administered telmisartan from week 8 to $20(n=5)$. ${ }^{*} \mathrm{P}<0.05$ compared to the Sham group. ${ }^{\dagger} \mathrm{P}<0.05$ compared to the MCA group.

improved liver fibrosis. Rats fed the MCA diet showed an increase in both HGF and TGF- $\$ 1$ production; however, administration of telmisartan resulted in an increase in HGF, 
but had no effect on TGF-ß1 production (Fig. 5). These results suggest that telmisartan regulates HGF, but not TGF- 11 production, leading to an anti-fibrotic action in this model.

\section{Discussion}

The present study demonstrated that telmisartan has an antifibrotic action in the improvement of NASH induced by an MCA diet. The beneficial effect of telmisartan might be due to an increase in HGF production in the liver. These properties are quite different from classical ARBs such as losartan (8). One possible explanation for the differential actions of telmisartan is its unique characteristic to stimulate PPAR- $\gamma$. In terms of cross-talk with HGF and PPAR- $\gamma$, it has been reported that PPAR $-\gamma$ binds to the putative peroxisome proliferator response element (PPRE) in the promoter region of the HGF gene, which leads to increased HGF gene transcription, mRNA expression and protein secretion. Although HGF shows multiple biological properties in various cells, including mitogenic, morphogenic and antiapoptotic activity, it also plays an important role in liver regeneration (9). Furthermore, it has been reported that HGF improves various types of hepatic fibrosis including the NASH model $(10,11)$, and HGF is also considered to be a downstream effector in the anti-fibrotic action of PPAR- $\gamma$ agonists (12). Thus, HGF is considered to be a downstream effector in the anti-fibrotic action of PPAR- $\gamma$ agonists (12).

During liver fibrogenesis, Ang II may promote TGF-ß1 expression and act as a pro-fibrogenetic factor through activation of hepatic satellite cells $(13,14)$. Thus, blockade of Ang II by telmisartan may have had an anti-fibrogenic action in this NASH model induced by the MCA diet. However, unexpectedly, the increased TGF- $B 1$ production was not attenuated by telmisartan in both of the co-treatment models. We speculate that the blockade of Ang II might not have been sufficient to improve liver fibrosis in this model. Indeed, there have been no successful reports involving this model using classical ARBs. In terms of HGF production, Ang II is known to be a potent negative regulator (15), and blockade of Ang II by an ARB might increase the local HGF concentration $(16,17)$. Interestingly, HGF is considered to be a downstream effector in the anti-fibrotic action of PPAR- $\gamma$ agonists (12). Thus, telmisartan may be a more potent inducer of HGF via PPAR- $\gamma$ activation, in addition to the blockade of Ang II. Indeed, our previous study found no increase in vascular HGF expression, different from telmisartan (8). The importance of PPAR $-\gamma$ activation is supported by the observation that the PPAR- $\gamma$ agonist, pioglitazone, markedly attenuated hepatic steatosis, inflammation and fibrosis in a rat model of NASH $(18,19)$.

Recent studies suggest that NASH, which is characterized by varying degrees of progressive steatosis, lobular inflammation and fibrosis of the liver, is related to insulin resistance and metabolic syndrome $(20,21)$. It is now recognized that patients with NASH present with a range of co-morbid conditions (e.g., diabetes, hypothyroidism and metabolic syndrome), and several studies have suggested that NAFLD is an independent cardiovascular risk factor that increases cardiovascular mortality $(22,23)$. In the development of NASH in a rat model, telmisartan as well as the
PPAR- $\gamma$ agonist, pioglitazone, markedly attenuated hepatic steatosis, inflammation, and fibrosis $(18,19)$. Telmisartan reduced the accumulation of visceral fat and decreased adipocyte size, to a much greater degree than did valsartan, with a reduction in hepatic triglyceride levels in rats (24). Thus, the pleiotropic effects of these drugs in the liver may have benefits in patients with metabolic syndrome and NASH. Overall, telmisartan has the potential to improve NASH induced by an MCA diet, possibly due to increased HGF production through its action as a partial agonist of PPAR- $\gamma$. These beneficial characteristics of telmisartan as a partial agonist of PPAR $-\gamma$ may provide a benefit in the treatment of metabolic syndrome beyond its blood pressure-lowering effect.

\section{Acknowledgements}

This study was supported by a Grant-in-Aid from the Ministry of Education, Culture, Sports, Science and Technology of Japan and by a Japan Heart Foundation Grant for Research. Telmisartan was obtained from Boehringer-Ingelheim.

\section{References}

1. De Gasparo M, Catt KJ, Inagami T, Wright JW and Unger T: International Union of Pharmacology. XXIII. The angiotensin II receptors. Pharmacol Rev 52: 415-472, 2000.

2. Benson SC, Pershadsingh HA, Ho CI, et al: Identification of telmisartan as a unique angiotensin II receptor antagonist with selective PPARgamma-modulating activity. Hypertension 43: 993-1002, 2004.

3. Schupp M, Janke J, Clasen R, Unger T and Kintscher U: Angiotensin type 1 receptor blockers induce peroxisome proliferator-activated receptor-gamma activity. Circulation 109: 2054-2057, 2004.

4. Lehmann JM, Moore LB, Smith-Oliver TA, Wilkison WO, Willson TM and Kliewer SA: An antidiabetic thiazolidinedione is a high affinity ligand for peroxisome proliferator-activated receptor gamma (PPAR gamma). J Biol Chem 270: 12953-12956, 1995.

5. Rosen ED and Spiegelman BM: PPARgamma: a nuclear regulator of metabolism, differentiation, and cell growth. J Biol Chem 276: 37731-37734, 2001.

6. Picard F and Auwerx J: PPAR(gamma) and glucose homeostasis. Annu Rev Nutr 22: 167-197, 2002.

7. Schupp M, Clemenz M, Gineste R, et al: Molecular characterization of new selective peroxisome proliferator-activated receptor gamma modulators with angiotensin receptor blocking activity. Diabetes 54: 3442-3452, 2005.

8. Nakagami H, Morishita R, Yamamoto K, et al: Hepatocyte growth factor prevents endothelial cell death through inhibition of bax translocation from cytosol to mitochondrial membrane. Diabetes 51: 2604-2611, 2002.

9. Michalopoulos GK and DeFrances MC: Liver regeneration. Science 276: 60-66, 1997.

10. Okano J, Shiota G and Kawasaki H: Protective action of hepatocyte growth factor for acute liver injury caused by D-galactosamine in transgenic mice. Hepatology 26: 1241-1249, 1997.

11. Kiyama S, Yamada T, Iwata H, et al: Reduction of fibrosis in a rat model of non-alcoholic steatohepatitis cirrhosis by human HGF gene transfection using electroporation. J Gastroenterol Hepatol 23: e471-e476, 2008.

12. Li Y, Wen X, Spataro BC, Hu K, Dai C and Liu Y: Hepatocyte growth factor is a downstream effector that mediates the antifibrotic action of peroxisome proliferator-activated receptorgamma agonists. J Am Soc Nephrol 17: 54-65, 2006.

13. Yoshiji H, Kuriyama S, Yoshii J, et al: Angiotensin-II type 1 receptor interaction is a major regulator for liver fibrosis development in rats. Hepatology 34: 745-750, 2001.

14. Matsuoka M, Zhang MY and Tsukamoto H: Sensitization of hepatic lipocytes by high-fat diet to stimulatory effects of Kupffer cell-derived factors: implication in alcoholic liver fibrogenesis. Hepatology 11: 173-182, 1990. 
15. Taniyama $\mathrm{Y}$, Morishita $\mathrm{R}, \mathrm{Nakagami} \mathrm{H}$, et al: Potential contribution of a novel antifibrotic factor, hepatocyte growth factor, to prevention of myocardial fibrosis by angiotensin II blockade in cardiomyopathic hamsters. Circulation 102: 246-252, 2000.

16. Nakano N, Moriguchi A, Morishita R, et al: Role of angiotensin II in the regulation of a novel vascular modulator, hepatocyte growth factor (HGF), in experimental hypertensive rats. Hypertension 30: 1448-1454, 1997.

17. Nakano N, Morishita R, Moriguchi A, et al: Negative regulation of local hepatocyte growth factor expression by angiotensin II and transforming growth factor-beta in blood vessels: potential role of HGF in cardiovascular disease. Hypertension 32: 444-451, 1998.

18. Fujita K, Yoneda M, Wada K, et al: Telmisartan, an angiotensin II type 1 receptor blocker, controls progress of nonalcoholic steatohepatitis in rats. Dig Dis Sci 52: 3455-3464, 2007.

19. Jin H, Yamamoto N, Uchida K, Terai S and Sakaida I: Telmisartan prevents hepatic fibrosis and enzyme-altered lesions in liver cirrhosis rat induced by a choline-deficient L-amino aciddefined diet. Biochem Biophys Res Commun 364: 801-807, 2007.
20. Angulo P: Nonalcoholic fatty liver disease. N Engl J Med 346: 1221-1231, 2002

21. Angulo P: Obesity and nonalcoholic fatty liver disease. Nutr Rev 65: S57-S63, 2007.

22. Quercioli A, Montecucco F and Mach F: Update on the treatments of non-alcoholic fatty liver disease (NAFLD). Cardiovasc Hematol Disord Drug Targets 9: 261-270, 2009.

23. Vuppalanchi R and Chalasani N: Nonalcoholic fatty liver disease and nonalcoholic steatohepatitis: Selected practical issues in their evaluation and management. Hepatology 49: 306-317, 2009.

24. Sugimoto K, Qi NR, Kazdova L, Pravenec M, Ogihara T and Kurtz TW: Telmisartan but not valsartan increases caloric expenditure and protects against weight gain and hepatic steatosis. Hypertension 47: 1003-1009, 2006. 